\title{
Negative Correlations between Control Performance and Verbalizable Knowledge; Indicators for Implicit Learning in Process Control Tasks?
}

\author{
Axel Buchner \\ University of Trier, Trier, Federal Republic of Germany \\ Joachim Funke \\ University of Bonn, Bonn, Federal Republic of Germany \\ Dianne C. Berry \\ University of Reading, Reading, UK
}

\begin{abstract}
Negative correlations between task performance in dynamic control tasks and verbalizable knowledge, as assessed by a post-task questionnaire, have been interpreted as dissociations that indicate two antagonistic modes of learning, one being "explicit", the other "implicit". This paper views the control tasks as finite-state automata and offers an alternative interpretation of these negative correlations. It is argued that "good controllers" observe fewer different state transitions and, consequently, can answer fewer post-task questions about system transitions than can "bad controllers". Two experiments demonstrate the validity of the argument by showing the predicted negative relationship between control performance and the number of explored state transitions, and the predicted positive relationship between the number of explored state transitions and questionnaire scores. However, the experiments also elucidate important boundary conditions for the critical effects. We discuss the implications of these findings, and of other problems arising from the process control paradigm, for conclusions about implicit versus explicit learning processes.
\end{abstract}

Implicit learning has been investigated in a number of experimental paradigms such as probability learning (e.g. Reber \& Millward, 1968), serial reaction time (e.g. Willingham, Nissen, \& Bullemer, 1989), anagram solving (e.g. Gardiner, Alison, \& Sutton, 1989), and

Requests for reprints should be sent to Axel Buchner, FB I-Psychologie, Universität Trier, Universitätsring 15, 54286 Trier, Germany. E-mail: buchner@cogpsy.uni-trier.de.

We would like to honour Donald E. Broadbent, who died during the preparation of this article. He contributed substantially to the development of this and other research conducted by the authors, and we are extremely grateful for having had the opportunity to interact and work with him.

The authors would like to thank Edgar Erdfelder for his valuable advice and support in performing the latent class analysis, and for helpful comments on an earlier draft of this paper. We also thank Peter A. Frensch, Robert C. Mathews, Stephen Monsell, Pierre Perruchet, and an anonymous reviewer for valuable comments, and we appreciate the help of Melanie Steffens and Rene Jerusalem for their assistance with data collection. 
grammar learning (e.g. Reber, 1967). The common claim in all these cases is that people learn more about structural properties of the material than they can verbalize.

In a recent review, Reber (1989) suggested that "similar observations concerning the exploitation of structure have been made in somewhat different contexts" (p. 221). He was referring to process control tasks for which it has been found that "despite the reasonably satisfactory performance which people could attain after practising this task, their ability to answer questions about the system was surprisingly poor" (Broadbent, 1977, pp. 193f). This dissociation between verbalization and control performance has been investigated intensively by Broadbent and his colleagues (e.g. Berry and Broadbent, 1984, 1987; Broadbent \& Aston, 1978; Broadbent, FitzGerald, \& Broadbent, 1986; Hayes \& Broadbent, 1988) and has led these researchers to conceptualize two different modes of learning: The so-called $S$-mode of learning is "selective, effortful, and reportable", and the $U$-mode is characterized by the "unselective and passive aggregation of information about the co-occurrence of environmental features and events" (Hayes \& Broadbent, 1988 , p. 251; see also Broadbent, 1989). The distinction between these two modes has also been equated with the distinction between explicit and implicit forms of learning (e.g. Berry \& Broadbent, 1988, p. 254).

In this paper, we discuss and illustrate some of the potential problems with finding a dissociation between performance in a dynamic control task and verbalizable knowledge about this task in a post-task questionnaire. In particular, we focus on the finding of negative correlations between task performance and the ability to answer specific questions correctly. Such a finding was reported by Berry and Broadbent (1984) and continues to be cited as evidence in favour of the explicit-implicit distinction in learning dynamic control tasks. The problem, we argue, is that the interpretation of these negative correlations in terms of the dissociated explicit and implicit learning processes may not be adequate in its present form. We present two experiments to support our arguments. Finally, we focus on some more general problems of the process control paradigm for conclusions about implicit versus explicit learning processes.

\section{The Sugar Factory as a Finite-state Automaton}

Berry and Broadbent (1984) and others (Berry, 1991; Marescaux, Luc, \& Karnas, 1989; McGeorge \& Burton, 1989; Squire \& Frambach, 1990; Stanley, Mathews, Buss, \& KotlerCope, 1989) used a simple computer-simulated scenario in which subjects control the production of sugar $(P)$ by manipulating the number of workers employed $(W)$. This "sugar factory" operates according to the following equation:

$$
P_{\mathrm{t}}=2 \times W_{\mathrm{t}}-P_{\mathrm{t}-1}
$$

where $1 \leqslant W \leqslant 12$ and $1 \leqslant P \leqslant 12$. The values of $W$ are multiplied by 100 and the values of $P$ are multiplied by 1000 to represent the number of workers and the sugar output in tons, respectively. According to the equation, the output of the system at time $t, P_{0}$ is determined completely by the last sugar output, $P_{\mathrm{t}-1}$, and the present input, $W_{\mathrm{t}}$, in terms of workers employed by the subject. (In addition, a "random component" is usually added such that on two-thirds of the trials the system changes, at time $t$, to a state that is one unit above or below the correct state according to the system equation.) 
For the present purposes, however, it will be more useful to think of this control task as a finite-state automaton. This alternative way of describing the task makes more obvious certain aspects of the learning process that we wish to emphasize. (For more detail on describing dynamic tasks as finite-state automata see Buchner \& Funke, 1993.) A finitestate automaton is specified by a finite set of input signals, a finite set of output signals, and a finite set of states. A transition function determines the next state of the system as a consequence of the input signal and the current state, and a result function determines the specific output signal of the system as a consequence of the state transition (Hopcroft \& Ullmann, 1979).

For the sugar factory, one can easily compute all cell entries (i.e. the states at time $t, P_{\mathrm{t}}$ ) using the system equation for all possible combinations of states $\left(P_{\mathrm{t}-1}\right)$ and inputs $\left(W_{\mathrm{t}}\right)$. The resulting $12 \times 12$ state transition matrix is given in Table 1 . To illustrate: if the system is in State 5 (fifth row in the matrix from the top; the last sugar output was 5000 tons), then input 8 ( 800 workers are employed) leads to State 11 (11,000 tons of sugar production).

\section{The Sampling Problem: Why Good Control May Result in Bad Verbalizable Knowledge}

One of the main results reported by Berry and Broadbent (1984) was the finding of generally negative correlations between control performance as assessed by the number of trials on target, and verbalizable knowledge as assessed by a post-task questionnaire.

TABLE 1

State Transition Matrix for the Sugar Factory

\begin{tabular}{|c|c|c|c|c|c|c|c|c|c|c|c|c|c|}
\hline \multirow{5}{*}{$\begin{array}{l}\text { Output } \\
\text { Signal: } \\
\text { Sugar }\end{array}$} & \multirow[b]{5}{*}{ Srate } & \multicolumn{12}{|c|}{ Input Signal } \\
\hline & & \multicolumn{12}{|c|}{ Workers } \\
\hline & & 100 & 200 & 300 & 400 & 500 & 600 & 700 & 800 & 900 & 1000 & 1100 & 1200 \\
\hline & & \multicolumn{12}{|c|}{ Input } \\
\hline & & 1 & 2 & 3 & 4 & 5 & 6 & 7 & 8 & 9 & 10 & $\|$ & 12 \\
\hline 1000 & 1 & 1 & 3 & 5 & 7 & 9 & 11 & 12 & 12 & 12 & 12 & 12 & 12 \\
\hline 2000 & 2 & 1 & 2 & 4 & 6 & 8 & 10 & 12 & 12 & 12 & 12 & 12 & 12 \\
\hline 3000 & 3 & 1 & 1 & 3 & 5 & 7 & 9 & 11 & 12 & 12 & 12 & 12 & 12 \\
\hline 4000 & 4 & 1 & 1 & 2 & 4 & 6 & 8 & 10 & 12 & 12 & 12 & 12 & 12 \\
\hline 5000 & 5 & 1 & 1 & 1 & 3 & 5 & 7 & 9 & 11 & 12 & 12 & 12 & 12 \\
\hline 6000 & 6 & 1 & 1 & 1 & 2 & 4 & 6 & 8 & 10 & 12 & 12 & 12 & 12 \\
\hline 7000 & 7 & 1 & 1 & 1 & 1 & 3 & 5 & 7 & 9 & 11 & 12 & 12 & 12 \\
\hline 8000 & 8 & 1 & 1 & 1 & 1 & 2 & 4 & 6 & 8 & 10 & 12 & 12 & 12 \\
\hline $9000^{*}$ & 9 & 1 & 1 & 1 & 1 & 1 & 3 & 5 & 7 & 9 & 11 & 12 & 12 \\
\hline 10,000 & 10 & 1 & 1 & 1 & 1 & 1 & 2 & 4 & 6 & 8 & 10 & 12 & 12 \\
\hline 11.000 & 11 & 1 & 1 & 1 & 1 & 1 & 1 & 3 & 5 & 7 & 9 & 11 & 12 \\
\hline 12,000 & 12 & 1 & 1 & 1 & 1 & 1 & 1 & 2 & 4 & 6 & 8 & 10 & 12 \\
\hline
\end{tabular}

* This state was defined as the target state of the system. However, States 8 and 10 were also counted as "target states".

Note: The cell entries represent the next state given a certain present state (sugar production, left column of the matrix) and a certain intervention (number of workers hired, top row of the matrix). 
The finding that "people who were better at controlling the tasks were significantly worse at answering the questions" has contributed to the speculation "that these tasks might, under certain conditions, be performed in some implicit manner" (Berry, 1993, p. 248), whereas the questionnaire data are assumed to reflect the results of explicit selective learning. The obvious implications of assuming two different learning processes to produce the negative correlations between control performance and questionnaire.performance are, of course, that these learning processes can be dissociated and, more importantly, that they can exert an inhibitory influence upon each other. In terms of the implicit-explicit learning distinction, the better one learns to control the task, the worse one learns to report verbally the structural features of the task, and vice versa (see e.g. Hayes \& Broadbent, 1988, p. 270).

There may, however, be an alternative explanation of these negative correlations that does not rely on postulating antagonistic implicit and explicit learning processes. Consider that Berry and Broadbent (1984, Experiment 1$)^{1}$ required their subjects to control the system from the very beginning of the experiment. "Good controllers" will, by definition, reach the target state more frequently than will "bad controllers". As a consequence, the "good controllers" will necessarily experience fewer transitions that are different from those leading to the target state. In other words, the section of the state transition matrix (see Table 1) covered by "good controllers" will be rather small. In contrast, "bad controllers" will not reach the target states very frequently. As a consequence, these subjects may experience a larger number of different state transitions. In other words, it is plausible that the "bad controllers" explore a larger section of the system's state transition matrix. Thus, the two types of subjects may have very different learning experiences. If these assumptions are reasonable, we should find a negative correlation between the number of different states experienced and the number of trials on target. It is, of course, possible for "bad controllers" merely to oscillate among a few different off-target states, in which case we would not expect a correlation different from zero.

Turning now to the post-task questionnaire administered by Berry and Broadbent (1984), we find that it contains items that probed subjects with a given work-force value, a given state of the system, and a next intervention. Subjects' task was to predict the next state of the system. For instance, subjects were told that the current work force was 600 , the current sugar output was 6000 tons, and the next work force would be 900 . Subjects tried to predict the resulting output from a given set of alternatives. Apart from providing the initial work-force value, the format of these items corresponds to that of (incomplete) state transitions in the system. The important point here is that the post-task questionnaire can be conceived of as a sample of state transitions from the matrix of state transitions of the sugar factory.

It follows rather directly from these considerations that subjects with a more restricted range of experiences with state transitions- "good controllers"-may have more difficulty in answering questions that are samples from the entire state-transition matrix. In contrast, "bad controllers" may find it easier to answer such questions, provided they really experience a larger number of different state transitions. On the basis of these

${ }^{1}$ Additional training and concurrent verbalization manipulations were applied in their Experiments 2 and 3, so these will not be considered here. 
considerations we hypothesized that a negative correlation between control task performance and performance on the questionnaire need not necessarily be explained in terms of antagonistic explicit and implicit learning mechanisms.

However, two restrictions apply to the argument put forward so far. First, we are not assuming that memorizing of state transitions is the only learning process relevant to process-control tasks. This would certainly be an oversimplification. For instance, subjects might use simple heuristics or mental models (Mathews et al., 1989; Stanley et al., 1989) for making interventions. We will consider this in greater detail in the discussion section (see also Buchner \& Funke, 1993). A second and related point is that the above predictions should hold true only for a specific stage of experience with the system. Consider, for instance, a person with only minimal experience with the task, or a person who learns very little, for whatever reason. We would expect this subject to show both very low control performance and also low performance on the post-task questionnaire. In contrast, a person who learns relatively fast and/or a person with extended experience might eventually learn a rule comparable to the equation normally used to communicate the sugar factory's system behaviour. As a result, this subject will be able to calculate the correct answers for the post-task questions as well as show very good control performance (see Sanderson, 1989). With these subjects, the two performance measures should correlate positively. It is between these extremes that we expect the specific pattern of correlations between the number of different state transitions on the one side and control performance as well as questionnaire scores on the other, the latter two of which should be correlated negatively.

To test our assumptions about the effects of the experienced state transitions, we performed a first experiment to replicate the results of Berry and Broadbent's (1984) Experiment 1. Two predictions follow from the above considerations: (1) We should be able to find a negative correlation between task performance and the number of different states experienced during the interaction with the system because "good controllers" should experience fewer different state transitions and "bad controllers" should experience a greater number of different state transitions. (2) The number of different states experienced should correlate positively with the number of questions answered correctly in the post-task questionnaire because these questions represent samples from the state transition matrix.

\section{EXPERIMENT 1}

\section{Method}

\section{Subjects}

Subjects were 38 ( 24 female and 14 male) University of Bonn students aged 18 to 45 years (median $=23$ ) who either volunteered or participated in the experiment for course credit. The number of subjects was chosen because a priori power considerations showed that under the assumption of $\alpha=\beta=0.05$, "large effects" (see e.g. Cohen, 1988) in correlations can be detected with the given sample size (Buchner, Faul, \& Erdfelder, 1992). Thus, $\alpha$ was set to 0.05 for all subsequent analyses, and individual $p$-values will be omitted. 


\section{Materials}

The Control Task. The control task corresponded closely to the original sugar factory, except that subjects did not use the keyboard to make inputs but the computer mouse. The display of the sugar factory is illustrated in Figure 1. Subjects clicked the " + " button to increase the work force by one step, and the "- "button to decrease it. They clicked "OK" when they were satisfied with their choice and then observed the consequences of their intervention. Both a numerical display of the last sugar production and a graph displaying the sugar output during the past interactions of the current block were presented. The sugar factory operated as illustrated in Table 1. On about two-thirds of all trials a "random component" was added such that the system changed to a state one above or below the correct state. The same sequence of random deviations from the deterministic model of the sugar factory was used for every subject. As in Berry and Broadbent (1984), States 8, 9, and 10 (corresponding to a sugar output of 8000,9000 , or 10,000 tons, respectively) were counted as being on target.

The Questionnaire. The post-task questionnaire was different from that used in the Berry and Broadbent study in the following ways: (1) We increased the number of questions that probed for state transitions to 12 ( 6 "simple transitions" and 6 "transition sequences", see further on). (2) We changed the response format for these multiple-choice questions such that all 12 possible system states were presented as response alternatives. An answer was scored as correct when the state selected as the response was either the correct state under the deterministic model or a neighbouring

\section{Dueruiew of sugar production}
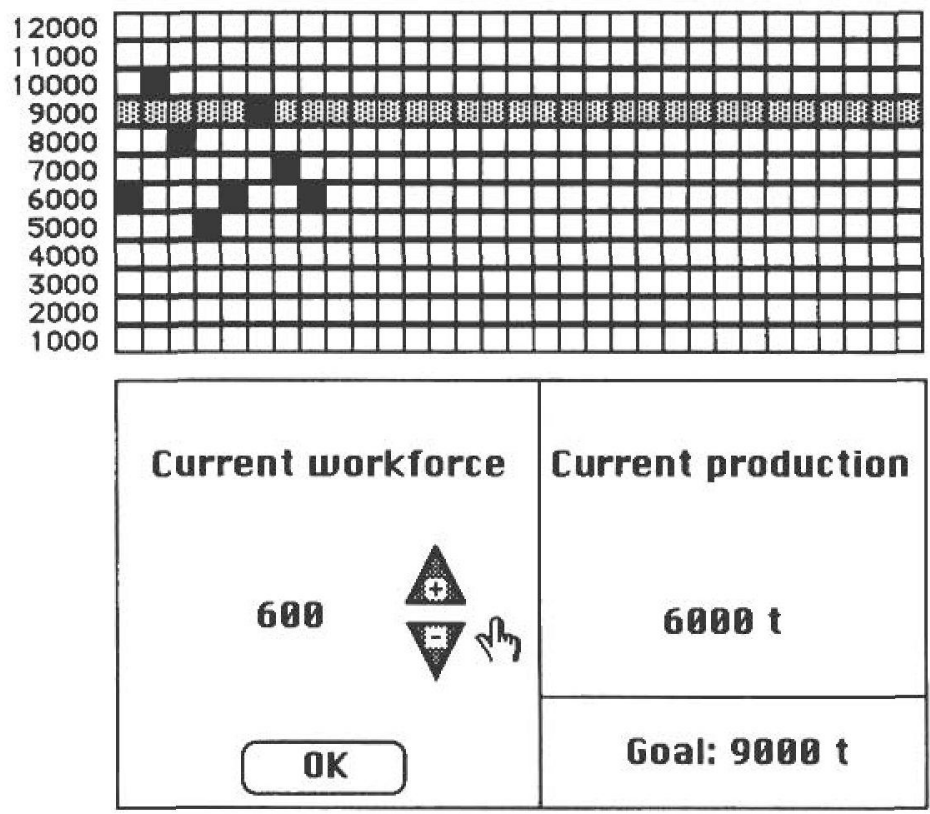

FIG. 1. Screen display of the sugar factory. Subjects increased or decreased the work force by clicking with the mouse on the "+" and "-" buttons, respectively. Clicking "OK" initiated the next trial. The resulting next state was displayed graphically by a black square in the trials graph and numerically under "current production". 
state. Scoring was thus identical for the control task and the questionnaire. (3) A further change concerned the initial work-force value, which was omitted for the six "simple transitions" questions that probed with a current state and an intervention for the next state.

As in Berry and Broadbent (1984), a second set of questions was used, which will be referred to as "transition sequences" questions. Subjects were given a sequence of three pairs of inputs and outputs, in both graphical and numerical form, designed in analogy to the sugar factory's screen display. Subjects were asked to make a numerical prediction about the next output given a new input. This question format is closer to the original task than that of the "simple transitions" questions and may thus provide a better "cue" to task-relevant knowledge. However, the transitions also contain system information that might perhaps be used by some subjects to modify their knowledge about the task during test. It seemed important, therefore, to compare performance in the "simple transitions" and "transition sequences" questions. The transitions addressed by the post-task questions are illustrated in the Appendix.

\section{Procedure}

The experiment was run on Apple Macintosh ${ }^{\mathrm{TM}} \mathrm{SE}$ microcomputers. Subjects were tested individually. Subjects' task was to interact with the system and to try to reach the target state ( 9000 tons of sugar output) as often as possible. Subjects had two blocks of 30 trials each to accomplish the task. They were not aware of the scoring scheme according to which outputs of 8000,9000 , and 10,000 tons of sugar were accepted as being on target.

The initial output was 6000 tons, and one "prior" intervention (600 work force) was initially visible on the screen. At this point, the post-task questionnaire was not mentioned. After the final intervention in the first block of 30 trials (Block 1) subjects were told that they had an additional block of 30 trials to reach the target (Block 2).

Subsequently, subjects were given the questionnaire to complete. It was stressed that they should apply what they had learned about the system. They were first given the 6 "simple transitions" questions. Upon completion of these questions they returned the questionnaire to the experimenter and received the questionnaire containing the 6 "transition sequences" questions.

\section{Results}

For Block 1, the scores for the number of trials on target ranged from 1 to 16 , with a mean of 7.6. On Block 2, these scores ranged from 3 to 19 , with a median of 9.1 . These scores were somewhat lower for both blocks than those reported by Berry and Broadbent (1984, Experiment 1). Also the increase from Block 1 to Block 2 was smaller, and it fell short of statistical significance, $t(37)=-1.60, p>0.12$. However, the ranges between the extremes seem comparable to those of the Berry and Broadbent study. The number of different state transitions during the control trials ranged from 9 to 28 on Block 1 and from 13 to 28 on Block 2. The means were 20.6 and 20.11 for Block 1 and Block 2, respectively.

For the post-task questionnaires, the mean scores for the "simple transitions" and for the "transition sequences" were 2.39 and 2.61 , respectively. These were not significantly different $(t<1)$. The hypothesis that subjects might learn from the information contained in the "transition sequences" questions was thus rejected, and the scores for both types of questions were combined for further analyses. 
Of central interest was the pattern of correlations between the number of different state transitions, the number of trials on target, and the questionnaire scores. As all hypotheses specified the direction of the correlations, one-tailed tests were used for statistical evaluation.

For Block 1, the Spearman rank correlation ${ }^{2}$ between the number of different state transitions and the trials on target was -0.03 . The correlation between the number of different state transitions and the questionnaire scores was 0.28 . The second, but not the first, correlation was significant, $t(36)=1.75$. The correlation between the trials on target and the final questionnaire scores was not significantly different from $0, r=0.01$.

For Block 2, we found a significantly negative correlation between the number of different state transitions and the trials on target, $r=-0.40, t(36)=-2.62$. The correlation between the number of different state transitions and the questionnaire scores was significantly positive, $r=0.37, t(36)=2.39$. The correlation between the trials on target and the questionnaire scores was negative, $r=-0.10$, but not significantly below 0 .

We further analysed whether subjects were better at post-task questions probing for states they had explored than for other questions. For each subject, we analysed the correct and incorrect answers to the questions to see whether the corresponding system states had been explored. The contingencies collapsed across all subjects are shown in Table 2. A prediction analysis (Hildebrand, Laing, \& Rosenthal, 1977) confirmed that there is a tendency for subjects to be better at answering questions about transitions they had experienced and vice versa. This tendency is statistically significant, $\nabla=0.11, z=$ 2.09 .

Interestingly, subjects who experienced more different states also took longer to complete an individual trial, $r=0.34, t(36)=2.17$, for Block 1 , and $r=0.44, t(36)=$ 2.94 , for Block 2 . In contrast, the time to complete a trial did not correlate significantly with the number of trials on target, $r=0.03$ for Block 1 , and $r=-0.09$ for Block 2 . Thus, subjects who were exposed during their interaction with the system to a greater diversity of state transitions also seem to have taken longer to contemplate this information. Subjects' control behaviour, however, did not covary with the time taken for the average trial.

\section{Discussion}

We found the predicted pattern of correlations between the number of different state transitions, and the trials on target as well as the questionnaire scores. These findings indicate that the better subjects are at controlling the system, the less they experience about it in terms of different state transitions. In contrast, subjects who, during their attempts to control the system, expose themselves to a larger diversity of state transitions, acquire more knowledge, which they can later report on the questionnaire. However, due to the variable pattern of interacting with the system, they also score lower on the trialson-target criterion.

\footnotetext{
${ }^{2}$ Spearman rank correlations were computed for greater comparability with the original study of Berry and Broadbent (1984). However, Pearson correlation coefficients yielded basically identical results.
} 
TABLE 2

Number of Questions Answered Correctly and Incorrectly in Experiment 1

\begin{tabular}{lrlrl}
\hline & \multicolumn{4}{c}{ Answers to Post-task Questions } \\
\cline { 2 - 5 } & \multicolumn{2}{c}{ Incorrect } & \multicolumn{1}{c}{ Correct } \\
\hline States not explored & 231 & $(220)$ & 146 & $(157)$ \\
States explored & 35 & $(46)$ & 44 & $(33)$ \\
\hline
\end{tabular}

Note: Expected counts are given in parentheses.

The negative correlation between task performance and questionnaire scores was not statistically significant. Similarly, Berry and Broadbent (1984) observed that although 9 out of 10 correlations between control performance and questionnaire scores across different experimental conditions were negative, only one of these and the average across all 10 correlations was significantly below zero. One possible reason for this finding is that the subject samples were heterogeneous in their course of learning. (For another example of the importance of different courses of learning in interaction with a very similar system, see Green \& Shanks, 1993.) As mentioned before, the specific pattern of a negative correlation between control performance and questionnaire scores is not expected for subjects who learn comparatively fast, or for those who, for some reason, learn and know very little about the task. Only for subjects between these states do we expect the pattern of correlations specified above.

\section{Mixture Distribution Analysis of Latent Subgroups}

Inspection of the raw data supported the assumption that there was a small number of subjects who showed both very good control behaviour on Block 2 and very high scores on the questionnaire. In addition, there appeared to be another small subset that showed rather low performance on both experimental tasks. Therefore, we performed a mixture distribution analysis on the scattergram of the trials on target and the questionnaire data. More precisely, we performed a latent moderator variable analysis (Erdfelder, 1988, 1990) which postulates that a given sample consists of several subpopulations or "latent classes". These latent classes can be conceived of as being the levels of a discrete latent moderator variable $Z$. The means, the standard deviations, and the relation between two variables $X$ and $Y$ may then depend on the level of $Z$ (i.e. on the latent class). In our case, variable $X$ refers to the questionnaire scores, and variable $Y$ refers to the trials on target. Assuming that $Z$ has $k$ levels $z_{\mathrm{j}}$ with $j=1, \ldots, k$, the probability density $f$ of the sample data $X=x$ and $Y=y$ results from the sum of the local probability densities within latent classes weighted by the sizes of the latent classes:

$$
f(X=x, Y=y)=\sum_{j=1}^{k} \mathrm{NV}\left(X=x, Y=y \mid Z=z_{j}\right) * \mathrm{p}\left(Z=z_{j}\right)
$$


The term NV ( $X=x, Y=y \mid Z=z_{j}$ ) denotes the bivariate normal density function within the $j$ th latent class. Note that this analysis has mostly heuristic value in the present context because the assumption of a bivariate normal distribution of the variables $X$ and $Y$ in each of the hypothesized latent subclasses may not be reasonable, due to the "discrete" nature of these variables. However, one may regard this assumption as an approximation to the true state of affairs. Using the LMA program (Erdfelder, .1989 ), we fitted separate models with four, three, and two classes to the data. Means $\left(\mu_{\mathrm{X}}\right.$ and $\left.\mu_{\mathrm{Y}}\right)$, standard deviations $\left(\sigma_{\mathrm{X}}\right.$ and $\left.\sigma_{\mathrm{Y}}\right)$, and correlations $\left(r_{\mathrm{XY}}\right)$ within each class were free parameters, as were the latent class proportions, $\mathrm{p}\left(Z=z_{j}\right)$. Note that the latent class analysis searches for parameter values that maximize the likelihood of the sample data for a given model (i.e. for the assumption of $k$ disjunct and exhaustive subclasses). It does not maximize any of the free parameters. LMA uses the EM-algorithm (Dempster, Laird, \& Rubin, 1977) to estimate these parameters of the local (class-specific) bivariate normal distribution. Only the model with two subclasses yielded a stable fit to the data. The analysis yielded for each individual the probability of belonging into each of the (in the present case: two) latent subclasses. Subjects were classified accordingly. Figure 2 illustrates the results of the latent moderator variable analysis and the classification. A total of 10 subjects fell into Class 1 ; the remaining 28 subjects fell into Class 2.

Our theoretical considerations and the empirical estimations of the latent moderator variable analysis appear to correspond quite well. The analysis separated the sample into two distinct groups. The first group contained all subjects that can be considered to represent the extreme states of either no learning or very good learning on the task for

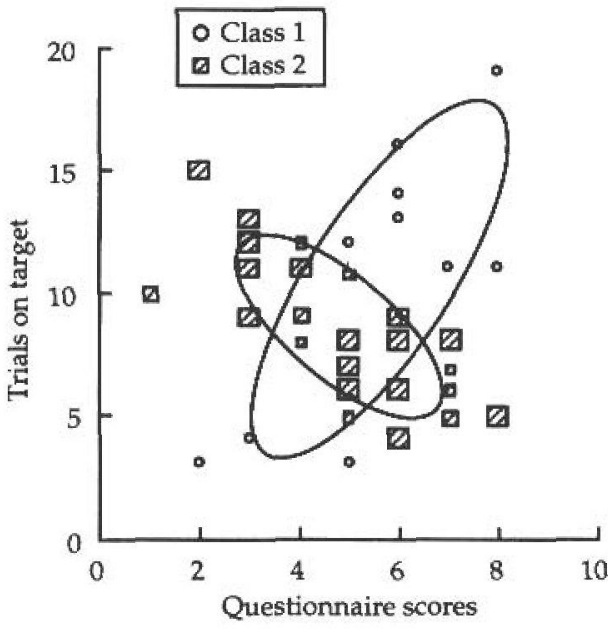

(a)

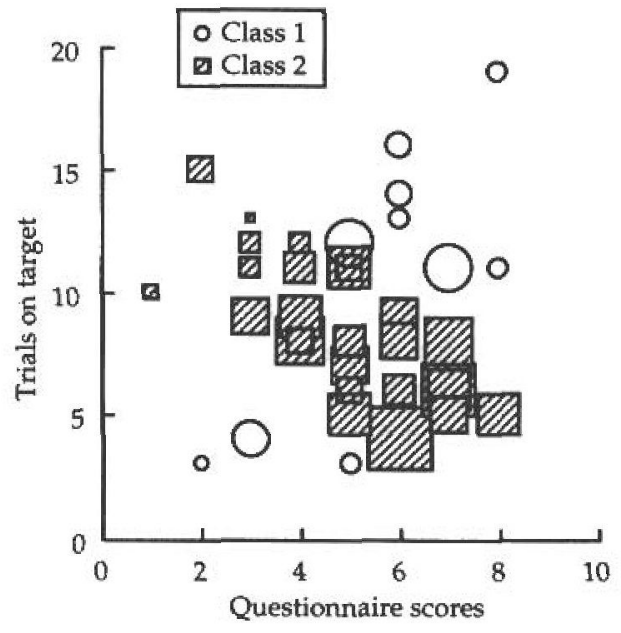

(b)

FIG. 2. (a) Distribution of subjects classified according to their control performance and their questionnaire scores into Class 1 (open circles) and Class 2 (filled squares) using a latent moderator variable analysis. The larger the symbols, the more subjects share one data point. The ellipses are centred on the sample means of both variables assuming a normal bivariate distribution of the data. (b) Same distribution as in the left part of the figure. The size of the symbols indicates the number of different state transitions experienced by each individual subject for Class 1 (open circles) and Class 2 (filled squares). 
which both control performance and questionnaire scores were very low or very high, respectively. The second group consisted of subjects ranging between these extremes. The latter group of subjects should exhibit a negative correlation between control performance and questionnaire scores. More importantly, only for these subjects do we expect a negative correlation between the number of different state transitions and the trials on target, and a positive correlation between the number of different state transitions and the questionnaire scores.

We performed two separate re-analyses to evaluate the critical correlational pattern within each subgroup. For Class 2 the correlation between control performance and questionnaire scores was significantly negative, $r=-0.73, t(26)=-5.45$. Much more interestingly, we found in Class 2 a significant negative correlation between the number of different state transitions and control performance, $r=-0.67, t(26)=-4.60$, and a significant positive correlation between the number of different state transitions and the post-task questionnaire scores, $r=0.64, t(26)=4.25$. Both correlations are higher than the correlations found for the entire sample. This is particularly important because the crucial variable for our argument, the number of different state transitions, was not a parameter in the latent moderator variable analysis. It is related to the remaining variables in a theoretically meaningful way, and this relation is confirmed by the analysis.

In contrast, for Class 1 neither the correlation between the number of different state transitions and task performance nor the correlation between the number of different state transitions and the post-task scores was significantly different from $0, r=0.09$ and $r=0.10$, respectively, and the correlation between both performance measures was significantly positive, $r=0.61, t(8)=2.18$.

In summary, the findings from Experiment 1 show that subjects' experiences with the system determine, in part, what is learned and, hence, what shows up in performance measures. A negative correlation between control performance and verbalizable knowledge may result because good controllers learn less about the transitions relevant to the post-task questions. However, this finding must be qualified in that the hypothesized pattern of correlations is present only for subjects in an intermediate state of learning about the system. The latter assumption is bolstered by the fact that for Block 1 the pattern was not observed. At this stage, presumably, most if not all subjects knew very little about the system.

Experiment 2 was designed to replicate and extend the findings from Experiment 1. Three different target states were used to see whether the results were influenced by the particular target state used. In addition, we used the three different target states to explore empirically the effect of manipulating how much of the state transition matrix subjects explore. One group of subjects, henceforth referred to as the variable group, had to reach a different target state on every new block of trials. In contrast, for the constant group, the target state remained the same. Across all blocks of trials, the variable group should experience more different state transitions than the constant group. If what subjects can report on the post-task questionnaire is a monotone function of the diversity of the learning experience (in terms of the number of different state transitions experienced), then the variable group should score higher on the post-task questionnaire. Further, in this case the critical pattern of correlations should be more pronounced for the variable group than for the constant group. 


\section{EXPERIMENT 2}

\section{Method}

\section{Subjects}

Subjects were 81 ( 57 female and 24 male) University of Bonn students aged 19 to 82 years (with a median of 22 years) who either volunteered or participated in the experiment for course credit. Given this sample size and $\alpha=\beta=0.05$, "large effects" (see e.g. Cohen, 1988) can be detected (1) between the two experimental groups (the variable group, 40 subjects, and the constant group, 41 subjects) and $(2)$ in correlations within each experimental group (Buchner et al., 1992). Thus, $\alpha$ was set to 0.05 for all subsequent analyses, and individual $p$-values will be omitted.

\section{Materials}

The Control Task. The control task was identical to that in Experiment 1, except that three different target states were defined instead of only one. The target states were 3,7 , and 10 (see Table 1), corresponding to a sugar output of 3000,7000 , and 10,000 tons. The scoring proceeded as in Experiment 1. For instance, if the goal was to produce 3000 tons of sugar, a sugar output of 2000 , 3000 , and 4000 tons was scored as being on target. The constant group subjects were instructed to reach the same target state in each of three blocks of 20 trials. Excluding two subjects with incomplete answers to the questionnaire, 13 subjects were assigned to each of target states 3,7 , and 10 . The 40 subjects in the variable group were assigned to a new target state from the same set of three states for each block. Six or seven subjects experienced each of the possible orders of target states.

The Questionnaire. The post-task questionnaire was identical to that used in Experiment 1, except that it was composed of 24 instead of 12 items, all of which were presented in the format of "transition sequences" questions (see Appendix).

\section{Procedure}

The procedure was identical to the one described for Experiment 1, except that subjects had available three blocks of 20 trials each to accomplish the task. In addition, subjects in the variable group were told that the target states would be different on each block of trials.

\section{Results}

There was no significant main effect of the set of target states on any of the dependent measures used, and no significant interaction with any other independent variables. Thus, for clarity of presentation this variable has been omitted from all subsequent analyses.

The number of different state transitions encountered was analysed to evaluate the effect of varying the number of target states experienced. As expected, a significant difference between the variable and constant groups was found with respect to the total number of different state transitions accumulated over all three trial blocks, $t(78)=-6.24$ (see Table 3).

The average number of target states reached during the three blocks of trials is also shown in Table 3. A $2 \times 3$ MANOVA, with type of target state (constant vs. variable) as 
TABLE 3

Performance Scores for Number of Trials on Target, Number of Correct Answers on the Post-task Questionnaire, and Total Number of Different State Transitions Experienced during System Control

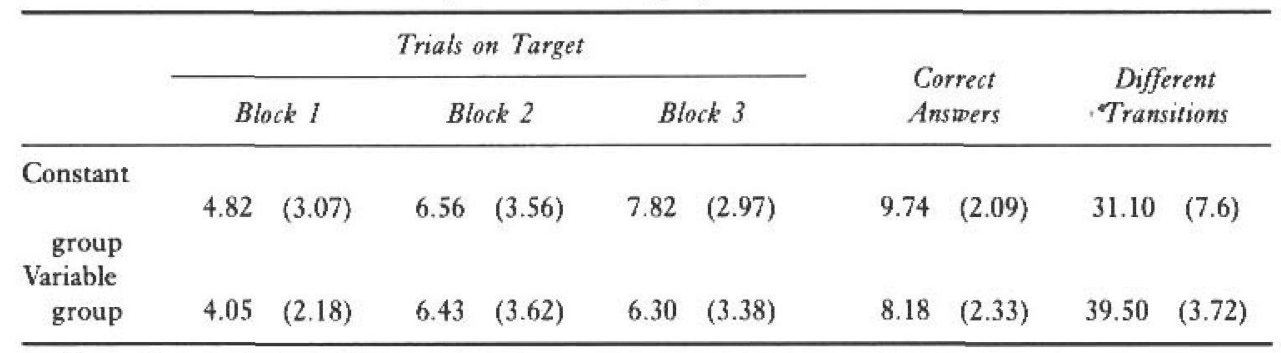

Note: Standard deviations are given in parentheses.

between-subjects variable and trial block $(1,2$ and 3$)$ as within-subjects variable, revealed only a significant effect of trial block, $F(2,76)=18.15$. Although at a descriptive level it appears that the constant group reached more target states than did the variable group, this difference just fell short of statistical significance, $F(1,77)=3.20, p=0.08$, and the Type of Target State $\times$ Trial Block interaction was also not significant, $F<1$. However, limiting the analysis to Block 3 as the final stage of the learning process in the present experiment, a two-tailed $t$-test yielded a significant advantage of the constant group over the variable group, $t(77)=2.16$. There was also a significant difference between constant and variable group with respect to the number of post-task questions answered correctly, $t(78)=3.15$, with the variable group actually answering fewer questions than the constant group (see Table 3). This result was not expected.

Looking now at the correlations involving the performance measures, the variables of interest are (1) the number of different state transitions accumulated across Blocks 1 to 3, which represents the entire system information encountered by a subject, (2) the number of trials on target in Block 3, and (3) the number of final post-task questions answered correctly immediately after completion of Block 3 (see Table 4).

TABLE 4

Correlations between Number of Different State Transitions, Number of Trials on Target, and Number of Correct Answers on the Post-task Questionnaire

\begin{tabular}{|c|c|c|c|c|c|c|}
\hline & & \multirow[b]{2}{*}{$\begin{array}{l}\text { Correct } \\
\text { Answers }\end{array}$} & \multicolumn{4}{|c|}{ Number of Different State Transitions } \\
\hline & & & $\begin{array}{c}\text { Blocks } 1-3 \\
\text { Combined }\end{array}$ & Block I & Block 2 & Block 3 \\
\hline \multirow[t]{2}{*}{ Constant group } & Trials on target & $-0.21^{\dagger}$ & $-0.47^{*}$ & -0.01 & $-0.55^{*}$ & $-0.59^{*}$ \\
\hline & Correct answers & & $0.33^{*}$ & 0.03 & 0.19 & 0.15 \\
\hline \multirow[t]{2}{*}{ Variable group } & Trials on target & $0.00^{\dagger}$ & $-0.20^{\dagger}$ & -0.03 & $-0.45^{*}$ & $-0.30^{*}$ \\
\hline & Correct answers & & -0.22 & $-0.28 *$ & $-0.39^{*}$ & -0.19 \\
\hline
\end{tabular}

$p<0.05$, one-tailed.

+ Number of trials on target in Block 3 was entered to compute these correlations. 
For the constant group, the correlation between the overall number of different state transitions and the trials on target was again significantly negative. The correlation between the number of different state transitions and the questionnaire scores was significantly positive, and, the correlation between the trials on target in Block 3 and the questionnaire score was negative. Although the latter correlation was larger than in Experiment 1, it was again not statistically significant.

The results for the variable group were quite different. (1) The correlation between the overall number of different state transitions and the trials on target was negative but not significantly different from 0 . Note, however, that for Blocks 2 and 3 the correlation between the number of different state transitions experienced mithin each block and the number of trials on target was significantly negative. (2) The correlation between the overall number of different state transitions and the questionnaire scores was negative but not significantly different from 0 (within Blocks 1 and 2 , the negative correlation was significantly different from 0 ), and (3) the correlation between the number of trials on target in Block 3 and the questionnaire score was zero.

We performed latent class analyses, fitting separate models with four, three, and two classes to the control performance and questionnaire data for both groups separately. None of these models fitted the data, suggesting that the two groups of Experiment 2 were not as heterogeneous as those of Experiment 1 (see Figure 3).

As in Experiment 1, we analysed whether subjects were better at post-task questions probing for states they had explored during their control trials than for other questions. The relevant data are shown in Table 5. A prediction analysis confirmed that, for the constant group, there is a statistically significant tendency for subjects to be better at answering questions about transitions they had experienced and vice versa, $\nabla=0.07, z=$ 2.07. In contrast, the variable group showed the reverse pattern-namely, a tendency to answer fewer questions about transitions they had experienced before than expected, $\nabla=$

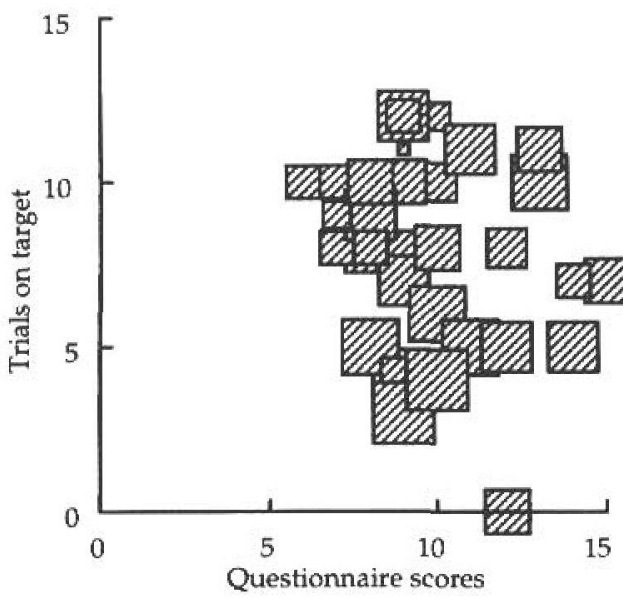

(a)

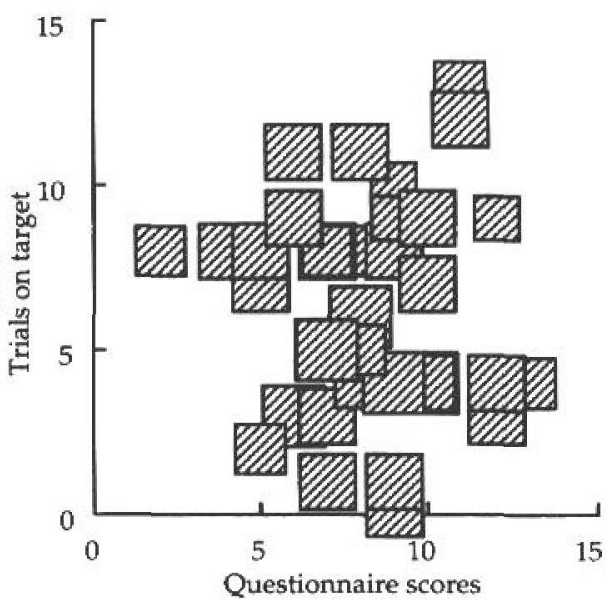

(b)

FIG. 3. Distribution of subjects of (a) the constant group and (b) the variable group. The size of the symbols indicates the number of different state transitions experienced by each individual subject. 
TABLE 5

Number of Questions Answered Correctly and Incorrectly in Experiment 2

\begin{tabular}{llllll}
\hline & \multicolumn{4}{c}{ Answers to Post-task Questions } \\
\cline { 2 - 6 } & \multicolumn{2}{c}{ Incorrect } & \multicolumn{2}{c}{ Correct } \\
\hline Constant group & & & & \\
$\quad$ States not explored & 428 & $(412)$ & 266 & $(282)$ \\
$\quad$ States explored & 128 & $(144)$ & 114 & $(98)$ \\
Variable group & 392 & $(415)$ & 238 & $(215)$ \\
$\quad$ States not explored & 241 & $(218)$ & 89 & $(112)$ \\
$\quad$ States explored & &
\end{tabular}

Note: Expected counts are given in parentheses.

$-0.11, z=-3.04$. This finding might seem surprising at first, but it has a very interesting basis. Our sample of state transition questions (see Appendix) contained three items that probed with an extreme intervention (1 or 12). These questions can be said to cover the "boundary conditions" of the system because the correct next state is simply the smallest or largest possible state ( 1 or 12), whatever the preceding state. As it turns out, the average proportions of "boundary condition" questions answered correctly relative to all correct answers by the variable group was much larger than that for the constant group: $(0.29$ vs. $0.40, t(76)=2.90)$. Thus, the variable subjects remembered much less about state transitions, but they knew the system boundaries relatively well. Knowing the boundaries implies that the next state can be determined by an extremely simple rule, so these subjects could answer many questions about transitions they had never seen, compared to the constant subjects.

\section{Discussion}

The constant group basically replicated the findings from Experiment 1 . The correlations involving the number of different state transitions were of comparable sizes in both experiments $(r=-0.40$ and $r=-0.47$ for trials on target in Experiments 1 and 2, respectively; $r=0.37$ and $r=0.33$ for the questionnaire scores in Experiments 1 and 2, respectively), and the correlation between the number of trials on target and the questionnaire scores was negative $(r=-0.10$ and $r=-0.21$ for Experiment 1 and 2, respectively). However, the latter correlations were not significantly below 0 , which raises the question as to whether the present experiments are true replications of the original Berry and Broadbent (1984) study. We are nevertheless confident that the present experiments are successful conceptual replications of the original study for at least two reasons: (1) The signs of the correlations between control performance and questionnaire scores were in the proper direction in both experiments. (2) Berry and Broadbent (1984) based their conclusion of an inverse relationship between control performance and questionnaire scores on an overall negative correlation obtained by averaging together 10 correlation coefficients from a number of different treatments. These correlation 
coefficients varied considerably (ranging from 0.21 to -0.80 , with an average of -0.31 ), and the present correlations seem well within that range.

The results for the variable group contradict our expectations. We had hypothesized that the chances to learn about the system and to report that knowledge on the post-task questionnaire would increase monotonically as a function of the number of state transitions to be explored. Instead, the requirement to reach a different target state on every block appears to have made the entire task much more difficult. This explanation is supported by the fact that the variable group was worse than the constant group at both control performance and questionnaire scores (see Table 3). It seems plausible to assume that, in contrast to the constant group, the variable group subjects had to explore too many single transitions to memorize them effectively and use this knowledge later for answering the post-task questions. In line with this assumption, we find consistent negative correlations between the number of different state transitions on the three trial blocks and the questionnaire scores for this group. Thus, extending the comparison between the constant and the variable groups, subjects within the variable group who were led to experience a particularly large number of different state transitions acquired even less knowledge appropriate for answering the post-task questions. The variable group, therefore, shows that knowledge reported on the post-task questionnaire is not a monotonic function of the diversity of system experience, and it provides further information about limiting conditions under which the critical correlational pattern is not found. Furthermore, the variable group also demonstrates why the learning of state transitions - although it may be important in explaining the negative correlations between control performance and questionnaire scores-is certainly not the only learning process involved in the present task. These subjects were relatively good at answering post-task questions that probed for the system's "boundary conditions", but with states subjects had not experienced before. Most probably, these questions were answered on the basis of a simple rule that was induced during subjects' exploratory experiences with these "boundary conditions" ("given the largest or smallest possible intervention, the largest or smallest possible state will result, respectively").

\section{GENERAL DISCUSSION}

Our present results suggest that one need not assume antagonistic explicit and implicit learning processes (e.g. Hayes \& Broadbent, 1988) to explain the inverse relationship between the number of trials on target and the questionnaire scores. (See also the conclusion reached by Green \& Shanks, 1993.) Rather, as has been demonstrated, this "dissociation" between task performance and verbalizable knowledge can result because of differences in subjects' experiences when interacting with the system. Most subjects with many trials on target have restricted experiences with the system. In contrast, subjects who are poor at controlling the system tend to collect experiences with a larger diversity of different state transitions. As a consequence, they are better at answering the post-task questions that probe for individual state transitions. Note that the present results bear only on the conclusions drawn from negative correlations between control performance and verbalizable knowledge. There is other evidence for dissociations in 
process control tasks on which our present findings have no bearing (c.g. Berry \& Broadbent, 1987, 1988; Broadbent et al., 1986).

Our assumption was that post-task questions can be answered on the basis of what can be remembered about state transitions while interacting with the system (see also Marescaux et al., 1989). One way this knowledge may be utilized is that certain state transitions are simply recognized on the questionnaire. Indeed, we observed a tendency for subjects to be better at answering post-task questions if the corresponding state transitions had been explored before than if they had not. However, subjects were also able to answer questions about states they had never explored. This can be explained if one assumes that state transition questions can be answered on the basis of "similarity matches"- that is, based on comparisons with similar remembered instances (Brooks, 1987; Jacoby \& Brooks, 1984). In either case the diversity of the learning experience will determine performance on the questionnaire, and the diversity of the learning experience should, as observed, be inversely related to the observed control performance.

The present experiments also elucidate some of the limiting conditions for this critical pattern of correlations. In Experiment 1, a latent moderator variable analysis confirmed our assumption that even if there exists a negative relation between control performance and questionnaire scores in a given sample of subjects, there may nevertheless be a subset of subjects for which this relationship does not hold. Basically, subjects who learn nothing while interacting with the system and subjects who learn both how to control the system and how to answer the questions could be separated from a majority of the sample ( $75 \%$ of the subjects) for whom the hypothesized pattern of correlations was even more pronounced than for the entire sample.

Furthermore, the variable group in Experiment 2 showed that when the number of different state transitions explored is increased by varying the target state between blocks of trials, then the influence of the diversity of system experience on the questionnaire scores may become detrimental while the negative relation between the diversity of system experience and control performance persists. Assuming that the memory trace representing a particular state transition is strengthened each time the transition is experienced, and assuming further that the trace strength must exceed a certain threshold before the transition can be retrieved, it is relatively easy to see that an increase in the number of different state transitions beyond a certain level may actually reduce the probability of retrieving certain state transitions (e.g. for answering the post-task questionnaire).

\section{How Many Learning Mechanisms?}

The present experiments offer an alternative to the assumption of antagonistic learning mechanisms for explaining the negative relation between control performance and questionnaire scores, but they do not speak directly to the question of hom many learning mechanisms should be assumed to explain the observed performance effects. From the rationale underlying the present research, a straightforward and certainly the most parsimonious assumption would postulate one single learning mechanism-namely the memorizing of state transitions. On each trial, a memory search is initiated based on the current system state and the target state as cues in order to retrieve an appropriate 
intervention or an intervention that belongs to a "similar" transition (Brooks, 1987; Jacoby \& Brooks, 1984). Although Stanley et al. (1989) did not use the term "state transitions", this corresponds exactly to what these researchers have called "memory for past experiences", or "close analogies", which, in their theory, is assumed to be the major basis for generating interventions. In terms of the Person Interaction Task used by Stanley et al. (1989), ${ }^{3}$ a memorized exemplar is "at least one prior Clegg response level plus the learner's response and Clegg's new response level" (p. 571) or, in other words, an output of the system at time $t-1$, an input at time $t$, and an output at time $t$. When answering the post-task questions, the situation is similar: A given system state and an intervention serve as cues for the next state that has to be predicted.

The first obvious problem with this simple explanation is the rule-like learning of boundary conditions that became apparent in Experiment 2. Furthermore, Stanley et al. (1989) argue that, in addition to developing memory for (lists of) state transitions, subjects incorporate "real world" mental models into the task. Mental models are assumed to be more accessible and easier to verbalize than state transition knowledge, which is why they should be preferred for verbal reports such as the instructions that Stanley et al. required their subjects to provide for another group of yoked subjects.

An example of a real-world mental model for the Person Interaction Task would be: "When you are talking to someone and you want them to be polite you should be polite to them ..." (Stanley et al., 1989, p. 572). This is a compact heuristic for making interventions. Interestingly, one can define heuristics or models that have no relation to the underlying structure of the system as defined by the experimenter but which nevertheless result in reasonably successful control performance. For instance, Stanley et al. (1989, Experiment 3) found that providing subjects with a simple heuristic for making interventions significantly facilitated control performance.

The post-task questionnaire used in Experiment 1 also contained an "open question" asking subjects to state how they went about solving the control problem. The answers to this question were so varied that it was impossible to evaluate them in any systematic way. However, we were able to identify five out of the 38 subjects who said it was important to keep the input at about 900 workers for an output of 9000 tons of sugar production. To illustrate the utility of such heuristics, we ran a simulation of 30 intervention trials with 5000 simulated subjects. As in the original study by Berry and Broadbent (1984), a different random influence was chosen for each individual subject (see also Berry, 1991; Marescaux et al., 1989; McGeorge \& Burton, 1989; Myers \& Conner, 1992; Squire \& Frambach, 1990; Stanley et al., 1989). For instance, one heuristic consisted of randomly choosing between a work force of 800,900 , or 1000 . The idea behind this is that a person could find it reasonable to employ a moderately, but not an extremely large, work force to obtain a moderately but not extremely large target output of 9000 tons within a range from 1000 to 12,000 tons. Trials on target ranged from 0 to 22 , with a mean of $9.76(S D=$ 3.44). A possible refinement of this heuristic would be consistently to employ a work force of 900 . One could choose to do this because a work force of 900 matches the desired output of 9000 tons in that it occupies the same ordinal position on the scale of possible inputs as does the target output value on the scale of possible outputs. With this strategy,

\footnotetext{
${ }^{3}$ This task is structurally equivalent to the sugar factory.
} 
trials on target ranged from 0 to 29 , with a mean of $10.34(S D=6.17)$. The success of these rather simple strategies that operate without ever taking the system behaviour into account is considerable. ${ }^{4}$

This leads to an important point. If subjects ever used such heuristics, they would not show up in the post-task questions probing for state transitions. This idea is similar to conclusions drawn by Perruchet and his colleagues for grammar learning, and serial reaction tasks (Perruchet \& Amorim, 1992; Perruchet, Gallego, \& Savy, 1990; Perruchet \& Pacteau, 1990). These authors have shown that dissociations between task performance indicating implicit processes and other measures indicating explicit processes can be explained by the fact that the knowledge assessed by the "explicit measures" was in fact irrelevant to the task performance. Similarly, in the present study, the good control performers can be said to have learned little that is relevant to answering the post-task questions. Rather, subjects' differential experiences with system state transitions can explain the negative correlations between control performance and questionnaire scores. Therefore, although no firm conclusion can be drawn about the number of learning mechanisms, the strong assumption of two antagonistic processes-one being explicit, the other being implicit-is certainly not necessary. Thus, the present research adds to the evidence that, before interpreting dissociations between measures of explicit and implicit processes, one must make sure that the same information is relevant to both types of measure.

\section{Implicit or Explicit Learning?}

As mentioned in the introduction, results obtained with control tasks such as the one considered in the present paper have, in the past, been related to conclusions drawn from observations in other implicit learning tasks. One reason to be more cautious about these comparisons is that the learning situation is deliberately kept incidental in typical implicit tasks such as grammar learning, anagram solving, or serial reaction-time tasks. In contrast, learning is always intentional in control tasks because subjects know from the beginning that they have to figure out the regularities in the system behaviour. Furthermore, in the performance test used in control tasks, the subjects' task is to predict the next system state given the current state and an intervention, knowing that they are to base their prediction on their past experiences of the regularities of the system behaviour. This performance test qualifies as a direct test of memory. In contrast, experiments on implicit tasks in which learning and performance testing are not separate (e.g. anagram solving, or serial reaction-time tasks) typically employ indirect tests of memory. Of course, it would be inappropriate to infer from the charactertistics of the task alone whether the underlying cognitive processes should be conceived of as either explicit or implicit. However, when the learning situation is intentional and the control performance test is direct, as in process control tasks, it seems a plausible assumption that explicit processes play a significant role.

From this perspective, the contribution of implicit processes would seem to be rather subtle. Like Stanley et al. (1989; see also Mathews et al., 1989) we assumed in this paper

\footnotetext{
${ }^{4}$ Also note that the range of trials on target that is solely due to the random component is very large.
} 
that subjects can base their interventions primarily on memory for past state transitions, at least under certain conditions. This requires that subjects search successfully in memory and retrieve a particular state transition as well as the proper intervention. If this explicit search fails, they might instead choose the intervention that "feels" most familiar given a particular system state. It is now received wisdom that the familiarity component can play a role in recognition memory (e.g. Gillund \& Shiffrin, 1984; Jacoby \& Dallas, 1981; Johnston, Dark, \& Jacoby, 1985; Mandler, 1980). In this sense, then, implicit influences might come into play. Originally Berry and Broadbent (1988) assumed "that if subjects adopt an implicit mode of learning they will build up in parallel large numbers of contingencies ... possibly . . . represented as a list of procedures or as some form of lookup table" (p. 271). In the light of the present evidence, the look-up table conception can be maintained, but the underlying learning processes are no longer characterized as being exclusively implicit.

\section{REFERENCES}

Berry, D.C. (1991). The role of action in implicit learning. Quarterly fournal of Experimental Psychology, $43 A, 881-906$.

Berry, D.C. (1993). Implicit learning: reflections and prospects. In A. Baddeley \& L. Weiskrantz (Eds.), Attention, selection, atpareness, and control: A tribute to Donald Broadbent (pp. 246-260). Oxford: Oxford University Press.

Berry, D.C., \& Broadbent, D.E. (1984). On the relationship between task performance and associated verbalizable knowledge. Quarterly Journal of Experimental Psychology, 36A, 209-231.

Berry, D.C., \& Broadbent, D.E. (1987). The combination of explicit and implicit learning processes in task control. Psychological Research, 49, 7-15.

Berry, D.C., \& Broadbent, D.E. (1988). Interactive tasks and the implicit-explicit distinction. British Journal of Psychology, 79, 251-272.

Broadbent, D.E. (1977). Levels, hierarchies, and the locus of control. Quarterly Journal of Experimental Psychology, 29, 181-201.

Broadbent, D.E. (1989). Lasting representations and temporary processes. In H.L. Roediger, \& F.I.M. Craik (Eds.), Varieties of memory and consciousness. Essays in honour of Endel Tulving (pp. 211-227). Hillsdale, NJ: Lawrence Erlbaum Associates, Inc.

Broadbent, D.E., \& Aston, B. (1978). Human control of a simulated economic system. Ergonomics, 21, $1035-1043$.

Broadbent, D.E., Fitzgerald, P., \& Broadbent, M.H.P. (1986). Implicit and explicit knowledge in the control of complex systems. British Journal of Psychology, 77, 33-50.

Brooks, L.R. (1987). Decentralized control of categorization: The role of prior processing episodes. In U. Neisser (Ed.), Concepts and conceptual development (pp. 141-174). Cambridge: Cambridge University Press.

Buchner, A., Faul, F., \& Erdfelder, E. (1992). G•Power: A priori, post-hoc, and compromise pomer analyses for the Macintosh [Computer program]. Bonn: Bonn University.

Buchner, A., \& Funke, J. (1993). Finite-state automata: Dynamic task environments in problem-solving research. Quarterly fournal of Experimental Psychology, 46A, 83-118.

Cohen, J. (1988). Statistical power analysis for the behavioral sciences (2nd ed.). Hillsdale, NJ: Lawrence Erlbaum Associates, Inc.

Dempster, A.P., Laird, N.M., \& Rubin, D.B. (1977). Maximum likelihood estimation from incomplete data via EM-algorithm. Journal of the Royal Statistical Society, B, 39, 1-22.

Erdfelder, E. (1988). Die Analyse latenter Moderatorvariablen [Analysis of latent moderator variables]. In W. Schönpflug (Ed.), Bericht über den 36, Kongreß der Deutschen Gesellschaft für Psychologie in Berlin, Band / (pp. 37-38). Göttingen: Hogrefe. 
Erdfelder, E. (1989). LMA: Analysis of latent moderator variables [Computer program]. Bonn: Bonn University.

Erdfelder, E. (1990). Deterministic developmental hypotheses, probabilistic rules of manifestation, and the analysis of finite mixture distributions. In A. von Eye (Ed.), Statistical methods in longitudinal research, Vol. 2: Time series and categorical longiudinal data (pp. 471-509). New York: Academic Press.

Gardiner, J.M., Alison, J.D., \& Sutton, E.A. (1989). Specificity and generality of enhanced priming effects for s self-generated study items. The American Journal of Pychology, 102, 295-395.

Gillund, G., \& Shiffrin, R.M. (1984). A retrieval model for both recognition and recall. Psychological Reviem, 91, 1-67.

Green, R.E.A., \& Shanks, D.R. (1993). On the existence of independent explicit and implicit learning systems: An examination of some evidence. Memory $\&$ Cognition, 21, 304-317.

Hayes, N.A., \& Broadbent, D.E. (1988). Two modes of learning for interactive tasks. Cognition, 28, 249-276.

Hildebrand, D.K., Laing, J.D., \& Rosenthal, H. (1977). Prediction analysis of cross classifications. New York: Wiley.

Hopcroft, J.E., \& Ullmann, S.D. (1979). Introduction to automata theory, languages, and computation. Reading, MA: Addison-Wesley.

Jacoby, L.L., \& Brooks, L.R. (1984). Non-analytic cognition: Memory, perception, and concept learning. In G.H. Bower (Ed.), The psychology of learning and motivation, Vol. 18 (pp. 1-47). Orlando, FL: Academic Press.

Jacoby, L.L. \& Dallas, M. (1981). On the relationship between autobiographical memory and perceptual learning. Journal of Experimental Psychology: General, 110, 306-340.

Johnston, W.A., Dark, V.J., \& Jacoby, L.L. (1985). Perceptual fluency and recognition judgments. Journal of Expermental Psychology: Learning, Memory, and Cognition, /1, 3-11.

Mandler, G. (1980). Recognizing: The judgment of previous occurrence. Psychological Review, 87, 252-271.

Marescaux, P.-J., Luc, F., \& Karnas, G. (1989). Modes d'apprentisage selective et non-selective et connaissances acquisés au controle d'un process: Evaluation d'un model simulé. Cahiers de Psychologie Cognitive European, 9, 239-264.

Mathews, R.C., Buss, R.R., Stanley, W.B., Blanchard-Fields, F, Cho, J.R., \& Druhan, B. (1989). Role of implicit and explicit processes in learning from examples: A synergistic effect. Journal of Experimental Psychology: Learning, Memory, and Cognition, 15, 1083-1100.

McGeorge, P., \& Burton, A.M. (1989). The effects of concurrent verbalization on performance in a dynamic systems task. British Journal of Psychology, 80, 455-465.

Myers, C., \& Conner, M. (1992). Age differences in skill acquisition and transfer in an implicit learning paradigm. Applied Cognitive Psychology, 6, 429-442.

Perruchet, P., \& Amorim, M.-A. (1992). Conscious knowledge and changes in performance in sequence learning: Evidence against dissociation. Journal of Experimental Psychology: Learning, Memory, and Cognition, 18, 785-800.

Perruchet, P., Gallego, J, \& Savy, I. (1990). A critical reappraisal of the evidence for unconscious abstraction of deterministic rules in complex experimental situations. Cognitive Psychology, 22, $493-516$.

Perruchet, P, \& Pacteau, C. (1990). Synthetic grammar learning: Implicit rule abstraction or explicit fragmentary knowledge? Journal of Experimental Psychology: General, 119, 264-275.

Reber, A.S. (1967). Implicit learning of an artificial grammar. Journal of Verbal Learning and Verbal Behaviour, 6, 855-863.

Reber, A.S. (1989). Implicit learning and tacit knowledge. Journal of Experimenal Psychology: General, IIS, 219-235.

Reber, A.S., \& Millward, R.B. (1968). Event observation in probability learning fournal of Experimental Psychology, 77, 317-327.

Sanderson, PM. (1989). Verbalizable knowledge and skilled task performance: Association, dissociation, and mental models. Journal of Experimental Psychology: Learning, Memory, and Cognition, 15, $729-747$. 
Squire, L.R., \& Frambach, M. (1990). Cognitive skill learning in amnesia. Psychobiology, 18, 109-117.

Stanley, W.B., Mathews, R.C., Buss, R.R., \& Kotler-Cope, S. (1989). Insight without awareness: On the interaction between verbalization, instruction, and practice in a simulated process control task. Quarterly fournal of Experimental Psychology, AIA, 553-577.

Willingham, D.B., Nissen, M.J., \& Bullemer, P. (1989). On the development of procedural knowledge. Journal of Experimental Psychology: Learning, Memory, and Cognition, 15, 1047-1060.

Revised manuscript received 9 January 1994

\section{APPENDIX \\ State Transitions Probed in the Post-task Questionnaire in Experiment 1 and in Experiment 2}

\begin{tabular}{|c|c|c|c|c|c|c|c|c|c|c|c|c|c|}
\hline \multirow{5}{*}{$\begin{array}{l}\text { Output } \\
\text { Signal: } \\
\text { Sugar }\end{array}$} & \multicolumn{13}{|c|}{ Input Signal } \\
\hline & \multirow[b]{4}{*}{ State } & \multicolumn{12}{|c|}{ Workers } \\
\hline & & 100 & 200 & 300 & 400 & 500 & 600 & 700 & 800 & 900 & 1000 & 1100 & 1200 \\
\hline & & \multicolumn{12}{|c|}{ Input } \\
\hline & & 1 & 2 & 3 & 4 & 5 & 6 & 7 & 8 & 9 & 10 & 11 & 12 \\
\hline 1000 & 1 & 1 & $3^{2}$ & 5 & $7^{b}$ & $9^{b}$ & 11 & 12 & 12 & 12 & 12 & 12 & 12 \\
\hline 2000 & 2 & 1 & $2^{b}$ & 4 & 6 & $8^{b}$ & 10 & 12 & 12 & 12 & 12 & 12 & 12 \\
\hline 3000 & 3 & 1 & 1 & 3 & $5^{a, b}$ & $7^{\mathrm{a}}$ & 9 & $11^{\mathrm{b}}$ & 12 & 12 & 12 & 12 & 12 \\
\hline 4000 & 4 & 1 & 1 & $2^{a, b}$ & 4 & $6^{\mathrm{b}}$ & 8 & 10 & 12 & 12 & 12 & 12 & 12 \\
\hline 5000 & 5 & 1 & 1 & 1 & 3 & 5 & $7^{b}$ & 9 & $11^{\mathrm{b}}$ & 12 & 12 & 12 & 12 \\
\hline 6000 & 6 & 1 & 1 & 1 & 2 & 4 & 6 & 8 & $10^{\mathrm{a}, \mathrm{b}}$ & $12^{2}$ & 12 & 12 & $12^{\mathrm{b}}$ \\
\hline 7000 & 7 & 1 & 1 & 1 & 1 & $3^{\mathrm{b}}$ & $5^{\mathrm{b}}$ & 7 & 9 & 11 & 12 & 12 & 12 \\
\hline 8000 & 8 & 1 & 1 & 1 & 1 & 2 & $4^{a, b}$ & $6^{a}$ & $8^{b}$ & 10 & 12 & 12 & 12 \\
\hline 9000 & 9 & 1 & $i$ & 1 & 1 & 1 & 3 & 5 & 7 & $9^{b}$ & 11 & $12^{2, b}$ & 12 \\
\hline 10,000 & 10 & $1^{\mathrm{b}}$ & 1 & 1 & 1 & 1 & 2 & 4 & $6^{b}$ & 8 & $10^{\mathrm{a}}$ & 12 & 12 \\
\hline 11,000 & 11 & 1 & 1 & 1 & $1^{b}$ & 1 & 1 & $3^{\mathrm{b}}$ & 5 & 7 & $9^{a}$ & 11 & 12 \\
\hline 12,000 & 12 & $1^{\mathrm{b}}$ & 1 & 1 & $1^{2}$ & 1 & 1 & 2 & $4^{b}$ & 6 & 8 & 10 & 12 \\
\hline
\end{tabular}

${ }^{2}$ Next states, Experiment 1.

${ }^{b}$ Next states, Experiment 2. 